Palavras chave:

E. dunnii

E. grandis

E. urophylla

Trabalhabilidade da madeira Acabamento superficial

Histórico:

Recebido 19/02/2010

Aceito 10/12/2013

Keywords:

E. dunnii

E. grandis

E. urophylla

Wood machinability

Roughness surface

Correspondência: camiladoubek@uel.br
Camila Santos Doubek Lopes', Adriana Maria Nolasco², Mário Tomazello Filho², Carlos Tadeu dos Santos Dias ${ }^{2}$

\section{AVALIAÇÃO DA RUGOSIDADE SUPERFICIAL DA MADEIRA DE Eucalyptus sp SUBMETIDA AO FRESAMENTO PERIFÉRICO}

RESUMO: Conduziu-se este trabalho, com o objetivo de avaliar a qualidade de superfície usinada da madeira de três espécies de Eucalyptus sp, para a produção de produtos madeireiros. Para os ensaios, foram confeccionados e usinados 48 corpos de prova de madeira de cada espécie, nos tratamentos de usinagem em fresadora de eixo vertical, no sentido discordante, em sete velocidades. $\mathrm{Na}$ avaliação da qualidade da superfície dos corpos de prova de madeira, utilizou-se o rugosímetro marca Taylor Hobson modelo Surtronic $3+$, utilizando o filtro $2 \mathrm{CR}$, com comprimento de amostragem com 0,80 le ou In $(\mathrm{mm})$ e cut-off de $0,80 \lambda c(\mathrm{~mm})$. Os resultados indicaram que a madeira do $E$. urophylla apresentou a melhor qualidade de superfície com os menores valores de coeficiente de variação; a madeira do $E$. grandis mostrou-se intermediária e a do $E$. dunnii apresentou o pior desempenho e o maior valor de coeficiente de variação. Observou-se, também, que o desempenho dos tratamentos para a madeira das espécies de Eucalyptus sp mostrou relação da redução da velocidade de avanço da usinagem com a rugosidade superficial; melhor na de 3-4 $\mathrm{m} \cdot \mathrm{min}^{-1}$ e pior na de $22 \mathrm{~m} \cdot \mathrm{min}^{-1}$. Constatou-se, também, que a qualidade da superfície da madeira usinada relacionou-se com a sua densidade e tipo de grã.

\section{EVALUATION OF WOOD SURFACE ROUGHNESS OF EUCALYPT SPECIES SUBMITTED TO CUTTERHEAD ROTATION}

ABSTRACT: This work evaluated the quality of roughness surface of 18 years-old Eucalyptus grandis, E. dunnii and $E$. urophylla trees wood, to produce prepared 48 furniture. For the laboratory essays samples/species were prepared in the machining treatments in milling machine of vertical axis, in the up milling direction, in seven cut speeds ( 3 to 22 $\left.\mathrm{m} \cdot \mathrm{min}^{-1}\right)$. In the evaluation of the surface quality of the wood samples it was used the surface profiling instrument Taylor Hobson model Surtronic $3+$, using the filter $2 \mathrm{CR}$, with sampling length of $0,80 \mathrm{le}$ or $\ln (\mathrm{mm})$ and cut-off of $0,80 \lambda \mathrm{c}(\mathrm{mm})$. The results indicated that the $E$. urophylla wood presented the best surface quality and with the smallest values of variation coefficient; the $E$. grandis wood showed intermediate values and $E$. dunnii wood presented the worst performance, as well as the largest value of variation coefficient. It was observed, also, that the treatment effect for the three eucalyptus wood species was related with the machining speed; better in the 3-4 $\mathrm{m} \cdot \mathrm{min}^{-1}$ and worse in the $22 \mathrm{~m} \cdot \mathrm{min}^{-1}$. The surface quality of the machined wood was related to its density, with the eucalyptus species of denser wood presenting the best quality of machined surface. 


\section{INTRODUÇÃO}

O potencial da madeira das espécies de eucalipto na fabricação de produtos sólidos vem sendo estudado no Brasil há algumas décadas. Nos últimos anos, verifica-se um aumento das pesquisas, visando a indicar as espécies potenciais de eucalipto para a produção de madeira de maior potencial, por meio da determinação das suas características anatômicas, físicas, químicas e mecânicas. Isso se deve, em grande parte, à necessidade de encontrar madeiras de plantações florestais que substituam às das espécies nativas, que apresentam restrições de exploração, escassez e custos elevados de transporte para os centros industriais e de consumo (OLIVEIRA, 1997).

Os estudos sobre a qualidade da superfície usinada, madeira de espécies de Eucalyptus, têm sido realizados no Brasil, por diversos autores (SILVA et al., 2005, 2007, 2009; SOUZA et al., 2009) indicando o potencial do gênero para a utilização da madeira para a produção de móveis, para uso sólido e de maior valor agregado. A otimização do processo de usinagem da madeira resulta em vantagens, como a diminuição do custo energético demandado pelas máquinas operatrizes, maior aproveitamento da madeira, aumento da vida útil das ferramentas e da produtividade e redução dos custos de usinagem e do produto final (SILVA, J. C., 2002).

A usinagem da madeira pode ser expressa pela função 5M, que compreende a (i) matéria-prima, relacionada com as propriedades intrínsecas da madeira, como a estrutura anatômica, a umidade e a grã; (ii) máquina, referente aos seus aspectos construtivos, como os dispositivos de fixação, condução e de exaustão dos cavacos; (iii) metodologia, que engloba a definição dos parâmetros de usinagem; (iv) mão de obra, considerando o treinamento dos operadores e o (v) meio ambiente, com as operações evitando a degradação do ambiente, objetivando o melhor aproveitamento da matéria-prima e minimizando os resíduos (BONDUELLE, 2003).

No processo de usinagem da madeira (Figura I) são produzidas pequenas ondulações na sua superfície da madeira, denominadas de avanço por dente $(f z)$. A medida do avanço por dente é resultado da interação entre a (i) velocidade de avanço (Vf) em $\mathrm{m} \cdot \mathrm{min}^{-1}$ correspondente ao movimento da peça de madeira e a ferramenta de corte; (ii) número de dentes ( $\mathrm{z}$ ) e a (iii) rotação $(n)$. $\mathrm{O}$ avanço por dente ( $\mathrm{fz}$ ) e a velocidade de avanço (Vf) são importantes parâmetros e inversamente proporcionais à qualidade da superfície da madeira, ou seja, quanto menores os valores de $f z$ e $V f$, melhor é a qualidade da superfície da peça de madeira. A velocidade de avanço $(V f)$ varia em função do

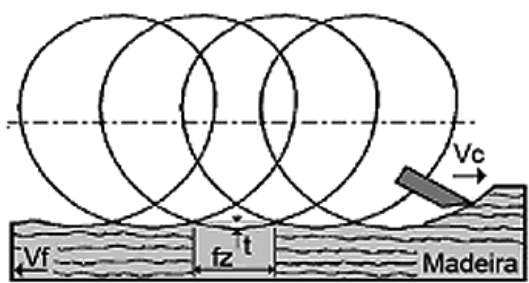

FIGURA I Grandezas de corte em aplainamento cilíndrico na direção discordante, sendo $f z$ (avanço por dente), $V c$ (velocidade de corte) e Vf (velocidade de avanço). Adaptado de Weissenstein (2000 apud SILVA, J. C., 2002).

FIGURE I Characteristics of cutterhead rotation: $\mathrm{fz}$ (feed per tooth), Vc (cut speed) and Vf (feedrate). Adap. Weissenstein (2000 apud SILVA, J. C., 2002).

tipo e da densidade da madeira, sendo que as de menor densidade são, normalmente, trabalhadas com uma maior velocidade de corte maior (SILVA et al., 2006).

Existem inúmeras técnicas de avaliação da qualidade da superfície da madeira usinada, como - apalpamento mecânico, ótica paramétrica e não paramétrica, microscopia, emissão acústica, ultrassom, pneumática, avaliação do tipo de cavaco, capacitância, laser, etc. (AGUILERA; MARTIN, 200 I; CASTRO, 2000). $\mathrm{Na}$ avaliação da qualidade de um perfil bidimensional da superfície da madeira, pode-se utilizar o rugosímetro, aplicado na análise da rugosidade e da superfície de metais, plásticos, etc., medindo a superfície segundo a variação vertical, em um deslocamento horizontal (SILVA et al., 2006). O valor da rugosidade (Figura 2) é função dos parâmetros da expressão matemática entre a variação vertical das superfícies (picos e vales) e o deslocamento horizontal do sensor (agulha), sendo os parâmetros divididos em 3 classes, em função da medida da profundidade da rugosidade, na horizontal e na proporcional (BONDUELLE, 2003).

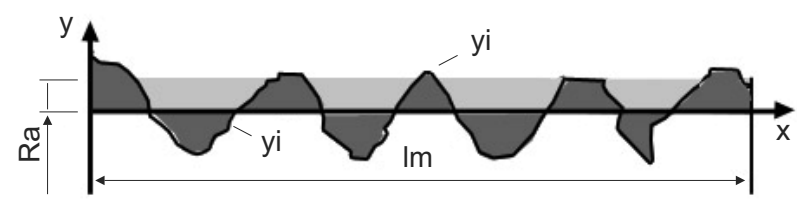

FIGURA 2 Representação do parâmetro $R a$, yi: ordenadas de afastamento; Im: percurso de medição. Fonte: Castro (2000).

FIGURE 2 Representation of the parameter Ra, yi: displacement coordinates; $\mathrm{Im}$ : reading area. Source: Castro (2000).

Em relação às espécies, verificou-se que a madeira do $E$. grandis apresentou o melhor acabamento de superfície em baixas velocidades de alimentação na 
operação de fresagem nos sentidos longitudinal ou paralelo às fibras, de acordo com Silva et al. (2006) que obtiveram melhor qualidade do acabamento superficial nas peças de madeira processadas a $15 \mathrm{~m} \cdot \mathrm{s}^{-1}$ em relação à de $30 \mathrm{~m} \cdot \mathrm{s}^{-1}$ de velocidade de avanço ( $\mathrm{Vf}$ ). Da mesma forma, o valor e a heterogeneidade da densidade ao longo da peça de madeira usinada das espécies de Eucalyptus, influenciam na rugosidade superficial (AGUILERA; MARTIN, 200I). Ainda, neste aspecto, Silva et al. (2009) atestaram que a redução de qualidade da superfície da peça de madeira está relacionada com a sua estrutura anatômica, pois, além de aumentar o avanço por dente, altas velocidades de avanço provocam maior arrancamento das fibras.

Pelo exposto, neste trabalho, objetivou-se analisar a rugosidade superficial da madeira de Eucalyptus grandis, $E$. dunnii e E. urophylla fresadas perifericamente em 7 velocidades de avanço nominais.

\section{MATERIAL E MÉTODOS}

\section{Espécies de Eucalyptus selecionadas}

Foram selecionadas 9 árvores de E. grandis, E. urophylla e E. dunnii (3 árvores/espécie, 18 anos) de plantações florestais da Estação Experimental do Departamento de Ciências Florestais da ESALQ/USP, localizada em Anhembi/SP (22 $47^{\circ}$ lat. S, $48^{\circ} 09^{\prime}$ long. W, $500 \mathrm{~m}$ alt., relevo suavemente ondulado e clima CWA).

\section{Amostragem e preparo dos corpos de prova de madeira}

Do tronco das árvores foram cortadas duas toras (4 $\mathrm{m}$ de comprimento) e desdobradas em pranchas tangenciais em serra de fita simples. As pranchas de madeira foram mantidas em área coberta e com ventilação natural para a secagem ao ar livre até atingir a umidade de equilíbrio de cerca de $12 \%$. Foram cortados 48 corpos de prova de madeira $(15 \times 3 \times 115 \mathrm{~cm}$; largura, espessura, comprimento) para cada espécie e, em seguida, realizadas 3 medições/corpo de prova com o rugosímetro em um total de 432 medições.

\section{Fresamento periférico dos corpos de prova de madeira}

Aplicou-se o fresamento cilíndrico periférico (Figura 3), compreendendo a usinagem dos corpos de prova de madeira em uma fresadora de eixo vertical (tupia), no sentido discordante, em 7 velocidades de avanço nominais

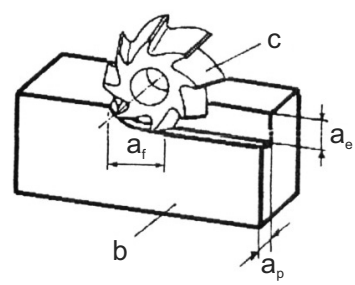

FIGURA 3 Fresamento cilíndrico periférico (b: peça de madeira; c: ferramenta de corte; $a_{p}$ : largura da usinagem; $a_{\mathrm{e}}$ : profundidade de corte; $\mathrm{a}_{\mathrm{f}}$ : penetração de avanço. Fonte: Castro (2000).

FIGURE 3 Milling operation (b: piece of wood; c: cut tool; $a_{p}$ : machining width; $a_{\mathrm{e}}$ : cut deep; $\mathrm{a}_{\mathrm{f}}$ : cut penetration. Source: Castro (2000).

$\left(3,4,6,8,11\right.$, 15 e $\left.22 \mathrm{~m} \cdot \mathrm{min}^{-1}\right)$. Na usinagem dos corpos de prova de madeira, a fresadora de eixo vertical (tupia moldureira) foi regulada a uma rotação de $3450 \mathrm{rpm}$, com cabeçote de fresa com faca de aço rápido de diâmetro de $92 \mathrm{~mm}$ e largura de $40 \mathrm{~mm}$, com ângulo de ataque ou saída $\gamma_{\mathrm{f}}=30^{\circ}$ e ângulo livre $\alpha_{\mathrm{f}}=10^{\circ}$, com dispositivo de avanço mecânico. A usinagem da superfície dos corpos de prova de madeira foi realizada no sentido paralelo às fibras $\left(90^{\circ}-0^{\circ}\right)$, com a aresta de corte em um ângulo de $90^{\circ} \mathrm{com}$ a direção das fibras e a família de corte a $0^{\circ} \mathrm{com}$ a direção das fibras. Na geração da superfície usinada dos corpos de prova de madeira, o dispositivo de avanço mecânico teve a função de manter a pressão constante e a velocidade pré-definida e assegurar o contato lateral total entre $\circ$ corpo de prova e a guia anterior.

A rotação do eixo da tupia moldureira foi mensurada com tacômetro manual, com 5 medições na aplicação do cálculo do valor médio de $5.814 \mathrm{rpm}$. Determinou-se, inicialmente, a velocidade do eixo $V c$ $\left(\mathrm{m} \cdot \mathrm{seg}^{-1}\right)$ da tupia moldureira, por meio da Equação I, sendo $d$ : diâmetro da ferramenta de corte $(\mathrm{mm})$ e $n$ : rotação do eixo da máquina (rpm).

$$
V c=\frac{\pi \times d \times n}{1000 \times 60}
$$

O avanço por dente $f_{z}(\mathrm{~mm})$, ou seja, a transição da peça-obra durante os sucessivos cortes foi expressa pela Equação 2, sendo $V f=$ velocidade de avanço $(\mathrm{mm}$. $\left.\min ^{-1}\right) ; z=$ número de dentes da ferramenta e $n=$ rotação do fuso (rpm).

$$
f z=\frac{V f}{Z \cdot n}
$$

$\mathrm{Na}$ operação de fresamento, a avaliação do desgaste da ferramenta de corte e a qualidade superficial são importantes, aplicando como indicador a qualidade do plano usinado, conforme adotado no trabalho. 


\section{Avaliação da rugosidade superficial da madeira}

Foi aplicada metodologia utilizada Castro (2000) e Gonçalves (1993), consistindo no apalpamento mecânico pelo rugosímetro Taylor Hobson, modelo Surtronic $3+$, com haste de medição com ponta de diamante, de forma cônica-esférica, raio de ponta de 2 e filtro $2 C R$. $O$ parâmetro determinado foi $\circ R a$ $(\mu \mathrm{m})$, utilizado na medição de superfície nos processos de fabricação de produtos da madeira e de outros materiais, constituído da média aritmética dos valores absolutos das ordenadas de afastamento (yi), dos pontos do perfil de rugosidade em relação à linha média, dentro do percurso de medição $(\mathrm{Im})$ (Tabela I) (GONÇALEZ et al., 2006).

\section{Análise estatística}

Efetuou-se, inicialmente, a análise exploratória dos dados de cada velocidade de avanço (teste de homogeneidade de variância outliers, escala e tamanho da amostra). Aplicou-se, em seguida, a análise da variância com a comparação das médias dos fatores e a interação das variáveis (teste de Tukey, nível de significância de 0,05$)$, considerando a madeira das 3 espécies de Eucalyptus e as 7 diferentes velocidades, em ensaio fatorial $3 \times 7$. Os dados foram analisados pelo sistema SAS versão 9.I.3, Service Pack 2, XP Plataform, 2002-2003 SAS Institute Corp. Cary, NC, USA.

\section{RESULTADOS E DISCUSSÃO}

Os resultados da aplicação das 7 velocidades de avanço na qualidade da superfície da madeira usinada (Tabela 2, Figura 4) indicam os menores valores médios de $R a$ para a madeira do $E$. urophylla, com diferença estatística entre as espécies, evidenciando um desempenho notadamente superior da qualidade da superfície. $O$ melhor resultado para a madeira do E. urophylla - de maior densidade $\left(0,69 \mathrm{~g} \cdot \mathrm{cm}^{-3}\right)$ corrobora o reportado por Aguilera e Martin (200I), Castro (2000), McKenzie et al. (200 I), Neri et al. (1999) e Silva et al. (2005) dentre outros. Por outro lado, os maiores valores médios de $R a$ relacionam-se com o pior desempenho da madeira do $E$. dunnii, de densidade $0,61 \mathrm{~g} \cdot \mathrm{cm}^{-3}$ e com as fibras formando a grã caracterizada como revessa. A disposição das fibras em grã revessa na madeira de espécies de Eucalyptus, a exemplo do $E$. grandis e do $E$. cloeziana, interfere no processo de usinagem e, dessa forma, afeta a qualidade da superfície da madeira, conforme destacam Castro (2000) e Gonçalez et al. (2006).

Nas velocidades de avanço de 3,8 e II $\mathrm{m} \cdot \mathrm{min}^{-1}$ não foram verificadas diferenças significativas na qualidade da superfície da madeira usinada das 3 espécies de Eucalyptus, com desempenho similar na velocidade de II m. $\mathrm{min}^{-1}$. Para o E. dunnii, os maiores Ra (pior desempenho de qualidade da superfície) foram obtidos a 3 e $8 \mathrm{~m} \cdot \mathrm{min}^{-1}$; para o E. grandis, os menores $R a$ (melhor qualidade da superfície) a 3 e $I$ I $\mathrm{m} \cdot \mathrm{min}^{-1}$ e para $\circ E$. urophylla, o menor e maior Ra (melhor e pior qualidade da superfície) a 8 e II $\mathrm{m} \cdot \mathrm{min}^{-1}$, respectivamente.

No entanto, foram verificadas diferenças na qualidade da superfície da madeira usinada das 3 espécies de Eucalyptus nas velocidades de avanço de 4, 6, I5 e $22 \mathrm{~m} \cdot \mathrm{min}^{-1}$. A madeira do $E$. dunnii mostrou o $R a$ mais elevado a 4,0 e $6,0 \mathrm{~m} \cdot \mathrm{min}^{-1}$ diferindo estatisticamente das 2 espécies; para o $E$. grandis o $R a$ mais alto foi de 15 e $22 \mathrm{~m} \cdot \mathrm{min}^{-1}$; para o E. urophylla, o menor Ra foi a 4, 6, I5 e $22 \mathrm{~m} \cdot \mathrm{min}^{-1}$, sendo similar ao do $E$. grandis.

Ainda, em termos médios, para a madeira das três espécies de Eucalyptus, as menores velocidades

TABELA I Parâmetros de velocidade do alimentador utilizados na rotação de 5.8।4 rpm.

TABLE I Applied parameters for rotation of $5.814 \mathrm{rpm}$.

\begin{tabular}{cccccc}
\hline $\begin{array}{c}\text { Velocidade } \\
\text { alimentador } \\
V c\left(\mathrm{~m} \cdot \mathrm{min}^{-1}\right)\end{array}$ & $\begin{array}{c}\text { Velocidade } \\
\text { efetiva } \\
V f\left(\mathrm{~m} \cdot \mathrm{min}^{-1}\right)\end{array}$ & $\begin{array}{c}\text { Avanço por dente } \\
f \mathrm{z}(\mathrm{mm})\end{array}$ & $\begin{array}{c}\text { Comprimento } \\
\text { de amostragem } \\
\text { le ou } \ln (\mathrm{mm})\end{array}$ & $\begin{array}{c}\text { Percurso } \\
\text { de medição } \\
\text { Im }(\mathrm{mm})\end{array}$ & $\begin{array}{c}\text { Cut-off } \\
\lambda c(\mathrm{~mm})\end{array}$ \\
\hline 3 & 2,53 & 0,43 & 0,80 & 4 & 0,80 \\
4 & 3,53 & 0,61 & 0,80 & 4 & 0,80 \\
6 & 5.53 & 0,95 & 0,80 & 4 & 0,80 \\
8 & 7,53 & 1,29 & 0,80 & 4 & 0,80 \\
11 & 10,53 & 1,81 & 0,80 & 4 & 0,80 \\
15 & 14,53 & 2,5 & 0,80 & 4 & 0,80 \\
22 & 21,53 & 3,7 & 0,80 & 4 & 0,80 \\
\hline
\end{tabular}


TABELA 2 Valores médios de densidade básica e de Ra para as 7 velocidades de avanço Vf para a madeira de Eucalyptus dunni, E. urophylla e E. grandis

TABLE 2 Average values of basic density and Ra for the 7 forward velocities $\mathrm{Vf}$ for Eucalyptus dunni, E. urophylla and $E$. grandis wood.

\begin{tabular}{|c|c|c|c|c|}
\hline \multirow{2}{*}{$\begin{array}{l}\text { Velocidade } \\
\left(\mathrm{m} \cdot \mathrm{min}^{-1}\right)\end{array}$} & \multicolumn{4}{|c|}{ Valores médios de $R a(\mu \mathrm{m})$} \\
\hline & E. dunnii & E. urophylla & E. grandis & Média \\
\hline $\begin{array}{l}\text { Densidade básica } \\
\qquad\left(\mathrm{g} \cdot \mathrm{cm}^{-3}\right)\end{array}$ & 0,61 & 0,69 & 0,58 & - \\
\hline$V f 3$ & 1,92 & 1,80 & 1,44 & 1,72 \\
\hline Tukey & $\mathrm{Ab}$ & $\mathrm{Ab}$ & $\mathrm{Ab}$ & c \\
\hline $\mathrm{CV}(\%)$ & 27,21 & 21,22 & 24,01 & 24,15 \\
\hline$V f 4$ & 2,90 & 1,61 & 2,04 & 2,19 \\
\hline Tukey & $\mathrm{Aa}$ & $\mathrm{BB}$ & $\mathrm{Bb}$ & $b$ \\
\hline CV(\%) & 27,41 & 27,25 & 26,96 & 27,20 \\
\hline$V f 6$ & 3,09 & 1,93 & 2,08 & 2,37 \\
\hline Tukey & $\mathrm{Aa}$ & BB & $\mathrm{Bb}$ & $b$ \\
\hline $\mathrm{CV}(\%)$ & 26,76 & 24,52 & 25,17 & 25,48 \\
\hline$V f 8$ & 2,61 & 1,96 & 2,09 & 2,22 \\
\hline Tukey & Aab & $\mathrm{Ab}$ & $\mathrm{Ab}$ & $b$ \\
\hline $\mathrm{CV}(\%)$ & 28,96 & 22,20 & 28,67 & 26,61 \\
\hline$V f I I$ & 2,29 & 2,45 & 2,08 & 2,27 \\
\hline Tukey & $\mathrm{Ab}$ & AA & $\mathrm{Ab}$ & $b$ \\
\hline $\mathrm{CV}(\%)$ & 25,11 & 35,26 & 36,34 & 32,24 \\
\hline$V f 15$ & 3,43 & 2,38 & 3,76 & 3,19 \\
\hline Tukey & $\mathrm{Aa}$ & $\mathrm{Bab}$ & $\mathrm{Aa}$ & a \\
\hline CV(\%) & 34,79 & 23,35 & 29,82 & 29,32 \\
\hline$V f 22$ & 3,46 & 2,87 & 4,19 & 3,50 \\
\hline Tukey & Aba & BA & $\mathrm{Aa}$ & $\mathrm{a}$ \\
\hline CV(\%) & 29,30 & 23,05 & 23,30 & - \\
\hline Média geral & 2,81 & 2,14 & 2,53 & - \\
\hline Tukey & $A$ & C & B & - \\
\hline $\mathrm{CV}(\%)$ médio & 27,75 & 28,50 & 25,26 & - \\
\hline
\end{tabular}

Médias seguidas da mesma letra - maiúscula na linha e minúscula na coluna - não diferem estatisticamente pelo teste de Tukey, com significância de $5 \%(p<0,05)$.

de avanço promoveram os menores valores de $R a$, indicando a melhor qualidade da superfície da madeira, com a rotação e o número de dentes da ferramenta mantidos constantes. A tendência de aumento dos valores de $R a$ com o da velocidade de avanço é concordante com a literatura (BONDUELLE, 2003; CASTRO, 2000; GONÇALVES, 2003), constatandose que a altura dos picos produzidos pela ferramenta aumenta exponencialmente com o avanço por dente $(f z)$

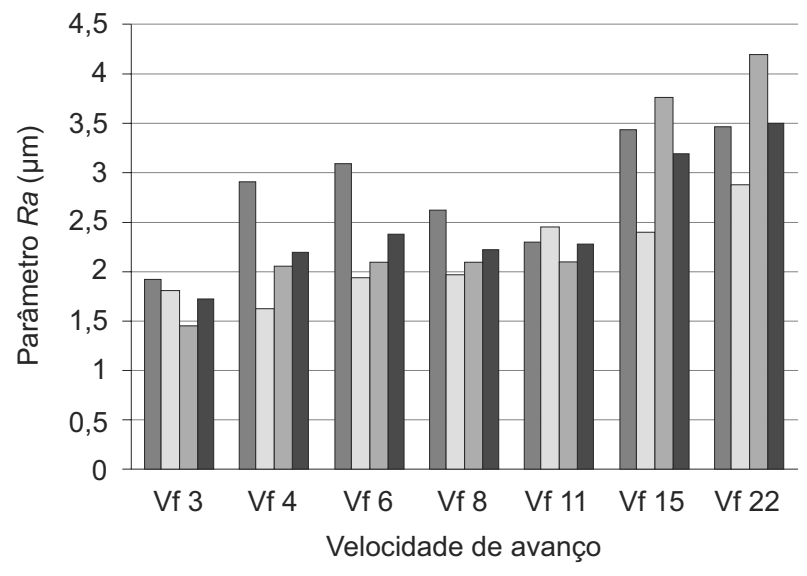

$\square$ E. dunnii $\square$ E. urophylla $\square$ E. grandis $\quad$ Média

FIGURA 4 Valores médios de Ra para cada velocidade de avanço e média geral para a madeira de E. dunnii, E. urophylla e E. grandis.

FIGURE 4 Average values of Ra for each forward velocity for E. dunnii, E. urophylla and $E$. grandis wood.

ou com a velocidade de avanço (Vf). Para a madeira do E. grandis e do Corymbia citriodora, as médias totais de $R a$ por $V f$ resultaram em significativa melhoria da rugosidade superficial das peças, proporcional à diminuição da Vf (GONÇALVES, 2003).

A madeira do $E$. dunnii apresentou valores de Coeficiente de Variação mais altos para todas as velocidades de avanço, exceto a II,0 $\mathrm{m} \cdot \mathrm{min}^{-1}$, indicando maior heterogeneidade das suas características, não recomendado no processo industrial. Os menores e maiores valores de $\mathrm{CV}$ foram obtidos na menor velocidade e a II $\mathrm{m} \cdot \mathrm{min}^{-1}$, embora, sem diferença para as médias da madeira das três espécies de Eucalyptus.

O lasqueamento, arrancamento e o arrepiamento das fibras na superfície da madeira usinada foram os principais defeitos observados nos ensaios laboratoriais. A madeira das espécies de Eucalyptus de menor densidade básica possui, normalmente, sua estrutura anatômica caracterizada por células de parede fina, de maior largura e diâmetro do lume e maior porcentagem de parênquima, que são danificadas por arrancamento na usinagem, resultando em rugosidade superficial grosseira e de baixa qualidade do acabamento (CASTRO, 2000; SILVA, J. R., 2002). Por outro lado, as espécies de madeira de maior densidade apresentam menor valor de rugosidade superficial, pela relação da densidade/resistência mecânica e pela dificuldade de penetração dos dentes do equipamento, influenciando no esforço de corte e acabamento final da superfície da madeira (AGUILERA; MARTIN, 200I; CASTRO, 2000; MCKENZIE et al., 200 I; NÉRI et al., 1999). 


\section{CONCLUSÕES}

Os resultados da avaliação da qualidade da superfície usinada da madeira das três espécies de Eucalyptus permitem concluir que: a qualidade da superfície da madeira usinada do E. urophylla, de maior densidade básica, foi superior com o aumento do avanço por dente (fz) e pela velocidade de avanço ( $\mathrm{Vf}$ ); os melhores resultados da qualidade da superfície da madeira usinada, indicam a espécie para a utilização em movelaria e na indústria de produtos de maior valor agregado; a madeira do $E$. dunnii, de menor densidade básica e fibras dispostas em grã revessa, apresentou o pior desempenho de qualidade da superfície usinada, mesmo com o aumento do avanço por dente $(\mathrm{fz})$ e da velocidade de avanço $(\mathrm{V} f)$; a madeira do $E$. grandis apresentou desempenho intermediário de qualidade da superfície usinada, mostrando visível melhoria com o aumento do avanço por dente $(f z)$ e da velocidade de avanço (Vf); e pelo valor médio obtido para a madeira das três espécies de Eucalyptus, verificase que a rugosidade superficial variou com o aumento do avanço por dente ( $f z)$, da velocidade de avanço $(V f)$ e com a densidade básica e o tipo de grã da madeira.

\section{REFÊRENCIAS}

AGUILERA, A.; MARTIN, P. Machining qualification of solid wood of Fagus silvatica and Picea excelsa: cutting forces, power requirements and surface roughness. Holz als Rohun Werkstoff, Berlin, v. 59, n. 6, p. 483-488, Dec. 200 I.

BONDUELLE, A. Processamento mecânico da madeira. Curitiba: UFPR, 2003.

CASTRO, E. M. Estudo da usinabilidade de chapas de MDF para usinagem de desbaste e acabamento. 2000. 122 p. Dissertação (Mestrado em Ciências e Engenharia dos Materiais) - Universidade de São Paulo, São Carlos, 2000.

GONÇALEZ, J. C.; BREDA, L. C. S.; BARROS, J. F. M.; MACEDO, D. G.; JANIN, G.; COSTA, A. F.; VALE, A. T. Características tecnológicas da madeira de Eucalyptus grandis e E. cloeziana visando ao seu aproveitamento na indústria moveleira. Ciência Florestal, Santa Maria, v. 16, n. 3, p. 329-34I, dez. 2006.

GONÇALVES, M. T. T. Proposta de metodologia para medição dos esforços de cortes na usinagem da madeira. 1993. I48 p. Tese (Doutorado em Ciências e Engenharia dos Materiais) - Universidade de São Paulo, São Carlos, 1993.
MCKENZIE, W. M.; CVITKOVIC, K. R.; RINGLER, M. Towards a model predicting cutting forces and surfaces quality in routing layered boards. Wood Science and Technology, Berlin, v. 35, p. 563-569, June 200 I.

NERI, A. C.; GONÇALVES, R.; HERNANDEZ, R. E. Forças de corte ortogonal 90-0 em três espécies de madeira de Eucalyptus. Revista Brasileira de Engenharia Agrícola e Ambiental, Campina Grande, v. 3, n. 2, p. 239-244, 1999.

OLIVEIRA, J. T. O. Caracterização da madeira de Eucalyptus para a construção civil. 1997. 428 p. Tese (Doutorado em Engenharia) - Universidade de São Paulo, São Paulo, 1997.

SILVA, J. C. Caracterização da madeira de Eucalyptus grandis de diferentes idades, visando sua utilização na indústria moveleira. 2002. 160 p. Tese (Doutorado em Tecnologia de Produtos Florestais) - Universidade Federal do Paraná, Curitiba, 2002.

SILVA, J. R. M. Relações da usinabilidade e aderência do verniz com as propriedades fundamentais do Eucalyptus grandis. 2002. 178 p. Tese (Doutorado em Tecnologia e Utilização de Produtos Florestais) Universidade Federal do Paraná, Curitiba, 2002.

SILVA, J. R. M.; LIMA, J. T.; BRAGA, P. P. C.; TRUGILHO, P. F. A utilização de rugosímetro na qualificação das superfícies usinadas em madeiras de Eucalyptus sp. In: ENCONTRO NACIONAL EM MADEIRAS E EM ESTRUTURAS DE MADEIRA, 10., 2006, São Pedro. Anais... São Carlos: EBRAMEM, 2006. I CD-ROM.

SILVA, J. R. M.; LIMA, J. T.; TRUGILHO, P. F. Usinabilidade da madeira de Eucalyptus grandis em diferentes regiões da medula à casca. Cerne, Lavras, v. 13, n. I, p. 25-31, jan./ mar. 2007.

SILVA, J. R. M.; MARTINS, M.; OLIVEIRA, G. M. V.; BRAGA, P. P. C. Parâmetros de qualidade da usinagem para a determinação dos diferentes usos da madeira de Eucalyptus. Cerne, Lavras, v. 15, n. I, p. 75-83, jan./mar. 2009.

SILVA, J. R. M.; MUÑIZ, G. I. B.; LIMA, J. T.; BONDUELLE, A. F. Influência da morfologia das fibras na usinabilidade da madeira de Eucalyptus grandis Hill ex. Maiden. Revista Árvore, Viçosa, v. 29, n. 3, p. 479-487, jun. 2005.

SOUZA, M. O. A.; SILVA, J. C.; LUCIA, R. M. D.; EVANGELISTA, W. V. Avaliação da madeira de Eucalyptus camaldulensis e Eucalyptus urophylla em ensaios de usinagem, visando à produção moveleira. Revista Árvore, Viçosa, v. 33, n. 4, p. 75 I-758, 2009. 